\title{
Stability and plasticity of silicon nanowires: the role of wire perimeter
}

\author{
J. F. Justo ${ }^{(1)}$, R. D. Menezes ${ }^{(1)}$, and L. V. C. Assali( ${ }^{(2)}$ \\ (1) Escola Politécnica, Universidade de São Paulo, \\ CP 61548, CEP 05424-970, São Paulo, SP, Brazil and \\ (2) Instituto de Física, Universidade de São Paulo, \\ CP 66318, CEP 05315-970, São Paulo, SP, Brazil
}

\begin{abstract}
We investigated the properties of stability and plasticity of silicon nanowires using molecular dynamics simulations. We considered nanowires with $\langle 100\rangle,\langle 110\rangle$ and $\langle 112\rangle$ growth directions with several diameters and surface facet configurations. We found that the wire perimeter, and not the wire diameter, is the meaningful dimensional parameter. As a result, the surface facets play a central role on the nanowire energy, that follows a universal scaling law. Additionally, we have computed the response of a silicon nanowire to external load. The results were compared to available experimental and $a b$ initio data.
\end{abstract}

PACS numbers: 61.46.-w, 68.65.-k 


\section{INTRODUCTION}

Silicon nanowires (SiNWs) belong to a unique class of semiconductor nanowires, since they may allow, in a near future, integration within conventional silicon-based device technology. In fact, the experimental realization of SiNW electronic devices has been achieved very recently [1]. Silicon nanowires have been synthesized by several methods [2, 3], generating wires in a wide range of diameters and crystallographic growth directions. Although the resulting wires are generally covered by oxide layers, complete removal of those oxide

layers has been achieved [4], leading to SiNWs with hydrogen passivated surfaces. SiNWs with reconstructed surfaces have also been produced [5].

SiNWs have been grown along several crystallographic directions: Holmes et al. reported SiNWs along the $\langle 100\rangle$ and $\langle 110\rangle$ directions with diameters of about $4 \mathrm{~nm}[3]$. Ma et al. reported ultra-thin SiNWs along the $\langle 100\rangle$ directions with diameters as low as $1.3 \mathrm{~nm}$ [4]. Wu et al. reported SiNWs along $\langle 110\rangle,\langle 111\rangle$, and $\langle 112\rangle$ lattice directions [6]. High resolution electron microscopy experiments have shown that the resulting SiNWs carry cores with monocrystalline bulk structures [4, 6].

Here, we carried a theoretical investigation on the stability of clean surface SiNWs along three growth directions and with a wide range of surface facet families. We found that the nanowire perimeter, and not its diameter, is the representative dimensional parameter to compute the wire scaling effects. The nanowire energy, for a certain growth direction, follows a universal scaling law with relation to the inverse of the nanowire perimeter. Additionally, we investigated the behavior of SiNWs under tensile deformation and compared the results with recent theoretical [7] and experimental data [8].

\section{METHODOLOGY}

Theoretical investigations, based on quantum mechanical calculations, have computed the properties of stable ultra-thin SiNWs [9-11]. However, such calculations are very expensive and investigations have been restricted to systems involving only a few hundred atoms and at an equilibrium state. Size-dependent and thermodynamical properties of nanowires are still unattainable to such methods. Although empirical methods carry a considerable simplification of the underlying atomistic processes, they still represent an alternative to 
access those important nanowire properties [7, 12].

Our simulations were performed using molecular dynamics, in which the Si-Si interactions were described by the Environment Dependent Interatomic Potential (EDIP) model \13, 14]. Some wire configurations were also computed using the Stillinger-Weber (SW) [15] and Tersoff [16] potentials, but the final results showed that the EDIP model provided the best description of silicon nanowires. The EDIP model [13, 14] includes environment terms that capture the essential elements of $\mathrm{Si}-\mathrm{Si}$ interactions in a wide range of atomic coordinations. That model represented a substantial improvement, as compared to previous models [15, 16], on the description of several important materials properties, such as disordered phases [17, 18], surfaces [14, 19], point [20] and line [21, 22] defects, and bulk elastic properties [23]. Therefore, this model appears to be an appropriate choice to investigate the stability and plasticity of SiNWs.

Depending on the wire configuration, the simulations involved up to 40,000 atoms. The wire stable configurations were computed by molecular dynamics, following an annealing procedure. The simulation of each wire started with a reasonably high temperature (600 $\mathrm{K}$ ), being slowly cooled down to a few kelvins, when the statistical properties were computed. We investigated the stability of SiNWs for $\langle 100\rangle,\langle 110\rangle$, and $\langle 112\rangle$ growth directions. Figure 1 shows a cross-sectional representation of the wires investigated here. For $\langle 100\rangle$ wires (fig. 17a), we considered five families with different facets, ranging from wires with pure $\{100\}$ surfaces to wires with pure $\{110\}$ surfaces. For $\langle 110\rangle$ wires (fig. 1b), we considered wire families ranging from prevailing $\{100\}$ to prevailing $\{111\}$ surfaces. Due to geometrical reasons, there is no $\langle 110\rangle$ wire comprising only $\{100\}$ or $\{111\}$ pure surfaces. For $\langle 112\rangle$ wires (fig. 1k), we considered wire families ranging from wires with prevailing $\{111\}$ surfaces to wires with prevailing $\{110\}$ surfaces. The facet configurations for those growth directions were consistent with images of $\langle 110\rangle$ and $\langle 112\rangle$ SiNWs [4]. Specifically in the case of the $\langle 112\rangle$ wires, the images revealed that the wires contained $\{111\}$ surfaces [4]. Additionally, the image intensity along the wire border suggested that those borders should be sharp. Considering those two elements, and that crystalline silicon $\{110\}$ surfaces have low energies, we assumed that the $\langle 112\rangle$ wires comprised only $\{111\}$ and $\{110\}$ surfaces. 


\section{RESULTS}

The scaling properties of nanowires have been described as a function of their diameters [8, 10, 11]. However, defining a nanowire diameter has been a challenging task, since nanostructures based on covalent bonding generally have facets and do not have a single diameter. Authors either avoid defining such a parameter [10, 12] or describe the wire representative dimension as its smallest diameter, taken from images of the wire cross-section [8]. Others take the diameter of the smallest cylinder that contains the wire [4, 11]. Ultimately, it is assumed that the nanowire has a prevailing cylindrical shape [11]. For large diameters, properties are reasonably well described using any of those assumptions, but not for thinner wires. Considering the SiNW faceting, we find that the wire perimeter $(P)$, and not the wire diameter, provides an appropriate description of the nanowire scaling properties. The wire perimeter comprises the sum of the length of each facet $\left(f_{i}\right)$ of the wire $\left(P=\sum f_{i}\right)$. The surface size of each facet is determined by $f_{i} \times L$ ( $L$ is the wire length) and the total wire surface is given by $P \times L$. As a result, a wire scaling law described in terms of its perimeter is equivalent to a law in terms of its total surface. Considering that in a nanowire, the surface/volume ratio is very large, it is reasonable to consider that scaling laws should be described in terms of the wire surface.

The nanowire total energy could be described by an analytical model, as recently discussed in ref. [24]. This energy $(F)$ comprises three elements: a bulk $\left(E_{b}\right)$, a surface $\left(E_{s}\right)$, and an edge $\left(E_{e}\right)$ term,

$$
F=E_{e}+E_{s}+E_{b},
$$

where the surface term is given by the contribution of all wire facets:

$$
E_{s}=\sum_{i} \gamma_{i} s_{i}
$$

where $\gamma_{i}$ is the surface energy of facet $i$, and $s_{i}$ is the number of unit cells in the surface [24].

The nanowire energy lies between two limits:

$$
E_{e}+\gamma_{\min } \sum_{i} s_{i}<\left(F-E_{b}\right)<E_{e}+\gamma_{\max } \sum_{i} s_{i}
$$

where $\gamma_{\min }$ and $\gamma_{\max }$ represent respectively the minimum and maximum values for the surface energy. Now, dividing all the terms by the number of atoms, $N \propto \mathrm{P}^{2}$, per unit length, one 
gets the following relation for the wire energy per atom (within some constant scale):

$$
E_{e} P^{-2}+\gamma_{\min } P^{-1}<\left(F-E_{b}\right) / N<E_{e} P^{-2}+\gamma_{\max } P^{-1}
$$

Equation 4 gives the limits for wire energy per atom, $\left(F-E_{b}\right) / N$, in terms of the wire perimeter. For large perimeters, the edge effects could be neglected, and the wire energy should have a linear relation with $P^{-1}$, and lie between two limiting cases, that are controlled by $\gamma_{\min }$ and $\gamma_{\max }$.

Figure 2 shows the energy per atom $\left(\mathrm{E}_{\mathrm{nw}}\right)$ of $\langle 100\rangle,\langle 110\rangle$, and $\langle 112\rangle$ SiNWs. This energy is defined with relation to the reference crystalline energy per atom, so that for very large wires, it tends to zero. We first consider the case of $\langle 100\rangle$ SiNWs. Using our classification in terms of wire perimeter, the nanowire energies follow a universal scaling law, for each facet family. The energy of a nanowire with any surface composition (pure or mixed character) falls within a certain region of the graphics, always between wires with $\{100\}$ and $\{110\}$ pure surfaces. These results would be expected: for a certain wire perimeter, the wire energy can have several values, depending on the surface types. The crystalline $\mathrm{Si}\{100\}$ surfaces have higher energies than $\{110\}$ surfaces [25], therefore it is consistent that wires (with the same perimeter) have higher energies if they have $\{100\}$ rather than $\{110\}$ pure surfaces. We performed additional calculations, considering wires with other compositions of $\{100\}$ and $\{110\}$ surfaces, between those two limiting cases of pure surfaces. For those cases, we found the wire energies lying inside that region. The figure also shows the results from $a b$ initio investigations [10], that had the dimensional parameters renormalized in order to describe energies in terms of wire perimeter. Our results for energies, in terms of interatomic potentials, of $\langle 100\rangle$ SiNWs are in good agreement with ab initio results.

For the $\langle 110\rangle$ SiNWs, we carried simulations for three facet families (fig. 1 b). Figure $2 \mathrm{~b}$ presents the energies of $\langle 110\rangle$ SiNWs as a function of the wire perimeter. The nanowire energies follows an equivalent scaling law as that of $\langle 100\rangle$ wires. Here, the wire energies fall within two limiting curves, which are related to wires with prevailing $\{100\}$ surfaces and wires with prevailing $\{111\}$ surfaces. The figure also shows the results from ab initio investigations [11]. In [11], SiNWs were constructed with a prevailing cylindrical shape of diameter $D$, so that the respective wire perimeters were well characterized by $P=\pi D$. Finally, figure $2 x$ presents the energies of $\langle 112\rangle$ SiNWs. Again, the energies are within two limiting lines, but here one of the limiting curves is related to nanowires with mixed 
character. According to fig. 2, our results are in in good agreement with ab initio data for all three growth directions.

Our results indicate that nanowire energies lie within two limiting energy lines, which are directly related to the character of the prevailing nanowire surfaces. Such results are fully consistent with the analytical model presented earlier. For large wire perimeters, edge effects can be neglected, and there is a linear relation between energy and the inverse of the wire perimeter. However, for smaller perimeters, edge effects become important [24, 26], which could explain the non-linear behavior of that relation. It should be pointed out that SiNWs with clean reconstructed surface are rare [5], while those with hydrogen passivated surfaces are more common [4]. There is a clear scaling behavior of wire energies as a function of wire perimeter, no matter if the surfaces are clean or passivated. If surfaces were passivated, the only difference would be the character of the energy limiting lines $\left(\gamma_{\max }\right.$ and $\left.\gamma_{\min }\right)$, which would be related to the energetics of those passivated surfaces.

We now discuss the properties of tensile deformation of $\langle 100\rangle$ SiNWs, with mixed $\{100\}+\{110\}$ facets. The deformation simulations were performed at constant temperature $(350 \mathrm{~K})$, by increasing the wire strains, followed by an equilibration process, and then computing the resulting uniaxial tensile stresses. Such simulation conditions tried to reproduce those of recent experiments on SiNW elasticity [8]. Figure 3 shows the stress-strain relation for SiNWs with several perimeters (11.0 nm, $13.2 \mathrm{~nm}$, and $16.9 \mathrm{~nm})$, along with the experimental data [8]. For small strains $(\varepsilon<0.05)$, the stress-strain curves have a linear behavior, which indicates an elastic response. For larger deformations $(0.05<\varepsilon<0.13)$, inelastic behavior takes place. At about $\varepsilon \approx 0.13$, there is a large decrease in the stress, for all wire perimeters, that is consistent with an equivalent behavior observed in another theoretical investigation [7]. The stress lowering is observed in experiments only for $\varepsilon \approx 0.25$, and indicated the fracture of the nanowire [8].

The stress-strain curves present a clear trend. For a certain strain, the wire stress depends strongly on the nanowire dimensions, a phenomenon that could be anticipated considering the dependence on the surface/volume ratio. The wire stress is the sum of the interatomic forces, normal to a certain cross-section area, divided by that area. Atoms in the wire surface are not fully coordinated, as those atoms in the wire core. As a result, they give only partial contribution to the wire stress. The experimental results, presented in the figure, correspond to deformations of ultra-thin nanowires [8]. Those authors assumed wires 
with a near cylindrical shape, and estimated the smallest wire cross-section of about 7.5 $\mathrm{nm}^{2}$, corresponding to a perimeter of about $10 \mathrm{~nm}$. Our results on deformation are in good agreement with experiments for small strains $(\varepsilon<0.13)$. However, the experimental data shows that wires support stronger deformations prior to collapsing. This discrepancy between theory and experiment may come from limitations of the theoretical potential in describing highly deformed materials or that the simulations were not performed in the adiabatic limit. On the other hand, according to fig. 3, the strain of collapse increased with the wire perimeter, in the direction of the experimental value. However, a direct comparison between theory and experiments is still difficult, since the wires used in those experiments were either hydrogenated or covered by a thin oxide layer while ours were reconstructed wires.

An interesting feature emerges from SiNW deformation simulations. It would be expected that, for a certain strain, the nanowire would simply follow a fracture process, as observed in experiments [8]. However, our simulations suggested a potentially richer phenomenology. The evolution of the nanowire deformation allows larger strains, with nanowire elongations considerably larger. The deformation process can be better understood by the wire evolution presented in fig. 4. For small strains (fig. 4a), the wire only elongates with an elastic response. For larger strains (fig. 4b), the wire starts to open a crack in the surface. However, instead of this crack just propagating along the wire, the wire becomes considerably thinner and continues elongating (figs. 4c and 4 d). The crack did not evolve because atoms in the surface had enough thermal energy to diffuse toward the crack, preventing crack propagation.

This mechanism of wire deformation could be useful in creating ultra-thin silicon nanowires for several applications, such as one-electron transistors. The process could be controlled by an atom force microscope operating at a certain temperature, in which the system had enough thermal energy to pump surface atoms toward the nanowire crack. However, this process would require an strict control over the applied forces, that we estimate in the order of only a few nano-Newtons.

\section{SUMMARY}

In summary, we found that the perimeter is the suitable dimensional parameter to describe the scaling properties of nanowires. The nanowire energies fall within two limit- 
ing cases, defined by the prevailing character of the wire surfaces. Considering the recent progress in growing SiNWs, these results provide additional elements to control the growing of nanowires not only with certain growth directions but also with certain surface facets. Recent investigations suggested that the surface facets are important in designing nanowires [27]. The surface electronic states may change the wire electronic properties, that is relevant for wire functionalization. For designing nanotransistors, one of the current challenges has been how to grow and manipulate ultra-thin nanoelements, for example with specific wire dimensions. The relevant effects of quantum confinement, and therefore gap engineering, is within a very strict region of wire diameters. Our results suggest that nano-mechanical processes, and not only chemical ones, may be used to design and create nanowires with specific properties.

\section{Acknowledgments}

The authors acknowledge support from the NANOSENSIM - Nanosensores Integrados e Microsistemas network (contract number 400619/2004-0 CNPq) and PNM (Programa Nacional de Microeletrônica). JFJ acknowledges partial support from brazilian agency FAPESP.

[1] Y. Cui, Z. H. Zhong, D. L. Wang, W. U. Wang, and C. M. Lieber, Nano Lett. 3, 149 (2003).

[2] A. M. Morales and C. M. Lieber, Science 279, 208 (1998).

[3] J. D. Holmes, K. P. Johnston, R. C. Doty, and B. A. Korgel, Science 287, 1471 (2000).

[4] D. D. D. Ma, C. S. Lee, F. C. K. Au, S. Y. Tong, and S. T. Lee, Science 299, 1874 (2003).

[5] B. Marsen and K. Sattler, Phys. Rev. B 60, 11593 (1999).

[6] Y. Wu, Y. Cui, L. Huynh, C. J. Barrelet, D. C. Bell, and C. M. Lieber, Nano Lett. 4, 433 (2004).

[7] M. Menon, D. Srivastava, I. Ponomareva, and L. A. Chernozatonskii, Phys. Rev. B 70, 125313 (2004).

[8] T. Kizuka, Y. Takatani, K. Asaka, and R. Yoshizaki, Phys. Rev. B 72, 035333 (2005).

[9] B.-X. Li, P.-L. Cao, R. Q. Zhang, and S. T. Lee, Phys. Rev. B 65, 125305 (2002). 
[10] R. Rurali and N. Lorente, Nanotechnology 16, S250 (2005).

[11] R. Kagimura, R. W. Nunes, and H. Chacham, Phys. Rev. Lett. 95, 115502 (2005).

[12] I. Ponomareva, M. Menon, D. Srivastava, and A. N. Andriotis, Phys. Rev. Lett. 95, 265502 (2005).

[13] M. Z. Bazant, E. Kaxiras, and J. F. Justo, Phys. Rev. B 56, 8542 (1997).

[14] J. F. Justo, M. Z. Bazant, E. Kaxiras, V. V. Bulatov, and S. Yip, Phys. Rev. B 58, 2539 (1998).

[15] F. H. Stillinger and T. A. Weber, Phys. Rev. B 31, 5262 (1985).

[16] J. Tersoff, Phys. Rev. B 38, 9902 (1988).

[17] L. Brambilla, L. Colombo, V. Rosato, and F. Cleri, Appl. Phys. Lett. 77, 2337 (2000).

[18] N. Mousseau N and G. T. Barkema, Phys. Rev. B 61, 1898 (2000).

[19] M. Prasad and T. Sinno, Appl. Phys. Lett. 80, 1951 (2002).

[20] G. Lulli, E. Albertazzi, S. Balboni, and L. Colombo, J. Phys.: Condens. Matter 18, 2077 (2006).

[21] J. F. Justo, V. V. Bulatov, and S. Yip, J. Appl. Phys. 86, 4249 (1999).

[22] J. F. Justo, M. de Koning, W. Cai, and V. V. Bulatov, Phys. Rev. Lett. 84, 2172 (2000).

[23] L. J. Porter, J. F. Justo, and S. Yip, J. Appl. Phys. 82, 5378 (1997).

[24] Y. Zhao and B. I. Yakobson, Phys. Rev. Lett. 91, 035501 (2003).

[25] A. A. Stekolnikov and F. Bechstedt, Phys. Rev. B 72, 125326 (2005).

[26] S. Ismail-Beigi and T. Arias, Phys. Rev. B 57, 11923 (1998).

[27] R. Rurali and N. Lorente, Phys. Rev. Lett. 94, 026805 (2005). 

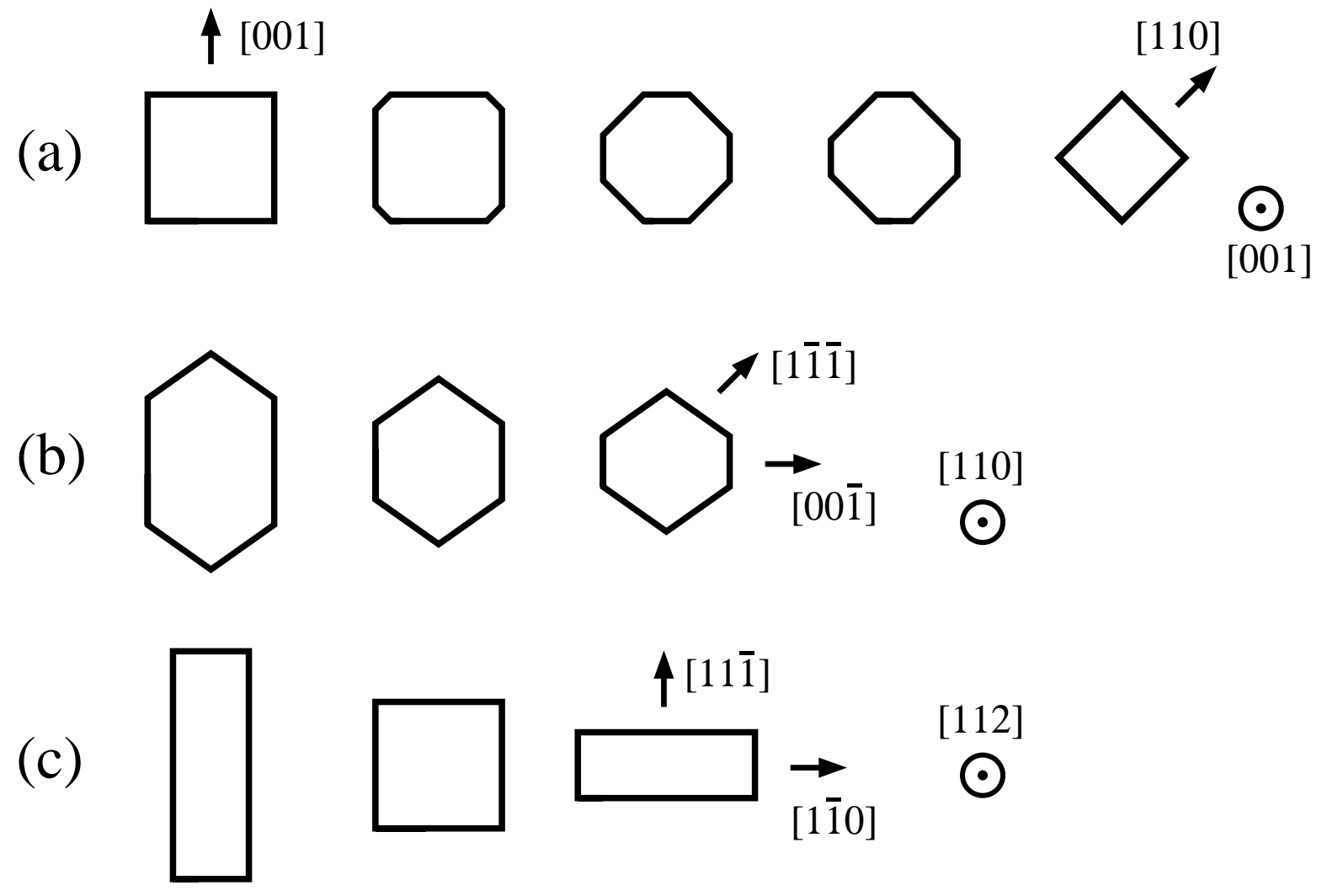

FIG. 1: SiNW configurations for different families in (a) $\langle 100\rangle$, (b) $\langle 110\rangle$, and (c) $\langle 112\rangle$ growth directions. For $\langle 100\rangle$ wires, we considered configurations ranging from pure $\{100\}$ surfaces to pure $\{110\}$ surfaces. For $\langle 110\rangle$ wires, we considered those ranging from prevailing $\{100\}$ surfaces to prevailing $\{111\}$ ones. For $\langle 112\rangle$ wires, we considered those ranging from prevailing $\{110\}$ surfaces to prevailing $\{111\}$ ones. 

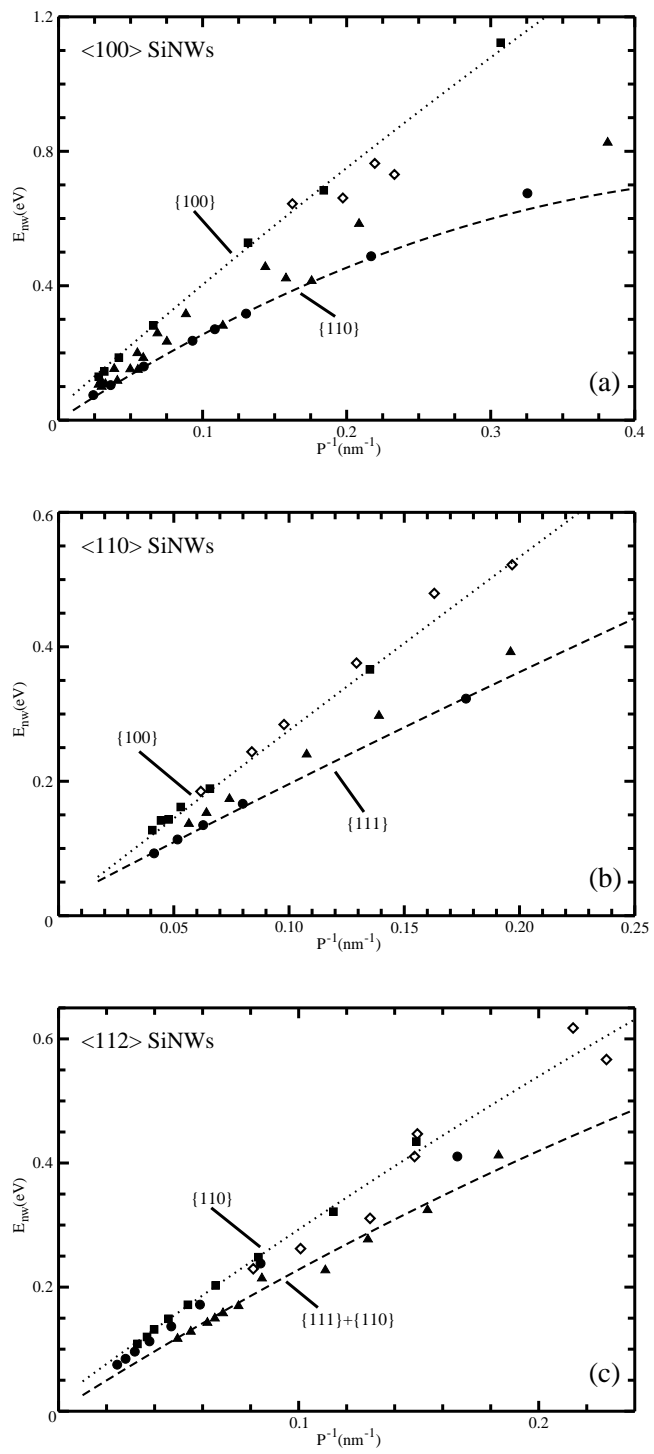

FIG. 2: Nanowire energy per atom $\left(\mathrm{E}_{\mathrm{nw}}\right)$ as function of perimeter for (a) $\langle 100\rangle$, (b) $\langle 110\rangle$, and (c) $\langle 112\rangle$ wires. Close symbols represent our results, while open ones the ab initio data: in (a) from ref. [10] and (b,c) from ref. [11]. In (a) the circles (•) represent wires with pure $\{110\}$ surfaces, the squares $(\boldsymbol{\square})$ those with pure $\{100\}$ surfaces, and the triangles $(\mathbf{\Lambda})$ those with mixed character. A few configurations with mixed character fell near the limiting case of wires with pure $\{110\}$ surfaces, but they comprised wires with prevailing $\{110\}$ surfaces. In (b) the circles $(\bullet)$ represent wires with prevailing $\{111\}$ surfaces, the squares ( $)$ those with prevailing $\{100\}$ surfaces, and the triangles $(\boldsymbol{\Lambda})$ those with mixed character. In (c) the circles $(\bullet)$ represent wires with prevailing $\{111\}$ surfaces, the squares (ם) those with prevailing $\{110\}$ surfaces, and the triangles $(\mathbf{\Delta})$ those with mixed character. The dotted and dashed lines in $(\mathrm{a}, \mathrm{b}, \mathrm{c})$ are data fittings coming from configurations that determine the energy limits. 


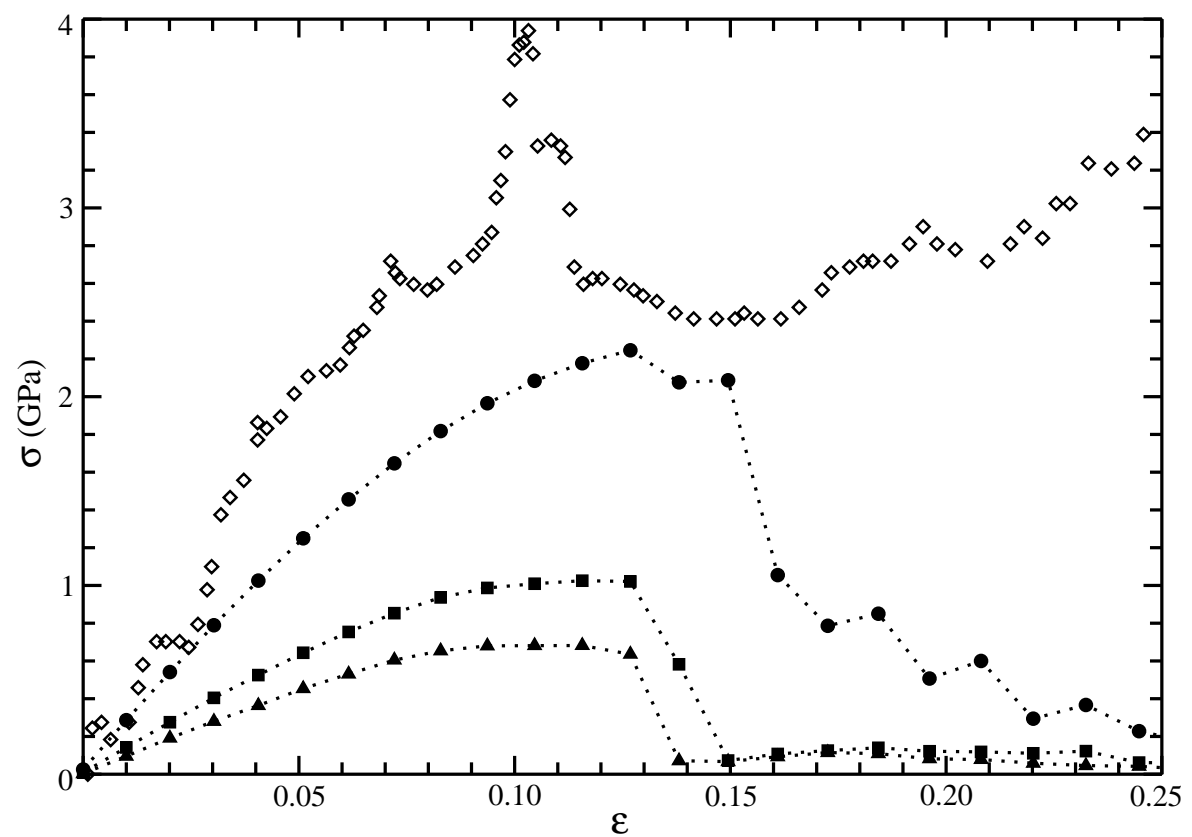

FIG. 3: Stress-strain curves as result of tensile deformation processes of $\langle 100\rangle$ SiNWs with mixed character $(\{100\}+\{110\})$. The close (open) symbols represent the results of our simulations (experiments). The figure shows the results for three different wire perimeters: triangles $(\boldsymbol{\Lambda})$, squares $(\boldsymbol{\square})$ and circles $(\bullet)$ represent respectively wires with perimeters of $11.0 \mathrm{~nm}, 13.2 \mathrm{~nm}$, and $16.9 \mathrm{~nm}$. 


\section{(a)}

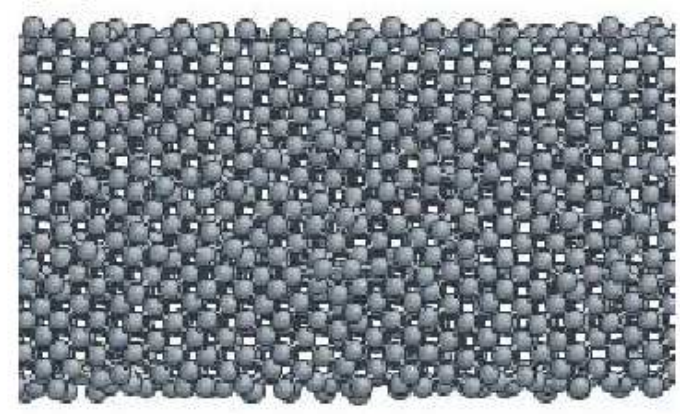

(c)

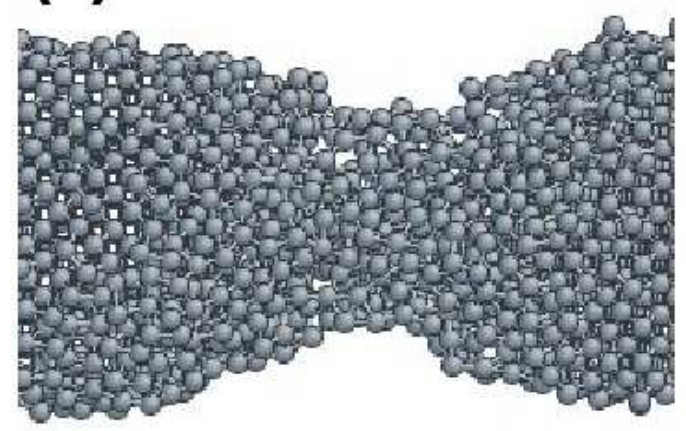

(b)

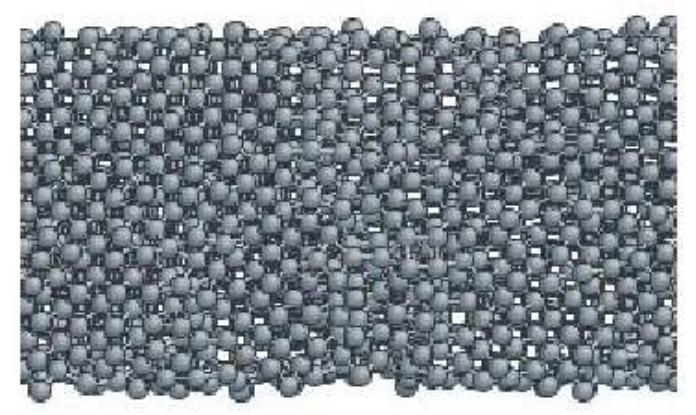

(d)

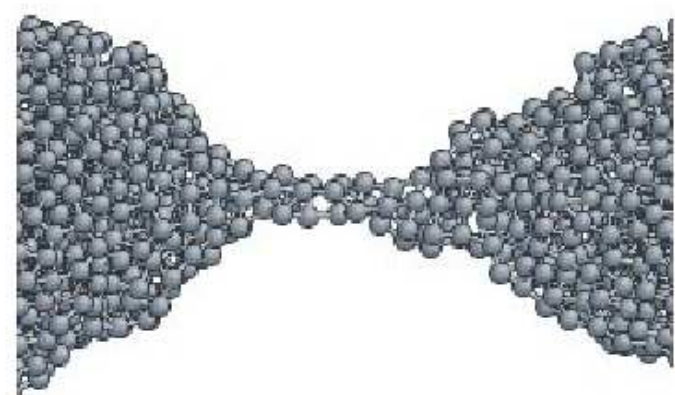

FIG. 4: Side view of the evolution of a $\mathrm{SiNW}$ (with a perimeter of $7 \mathrm{~nm}$ ) as result of external strain. The snap-shots correspond to configuration under different strains: (a) 0.07, (b) 0.08, (c) 0.10, and (d) 0.19. 